\title{
Direct Determination of Large Spin-Torque Nonadiabaticity in Vortex Core Dynamics
}

\author{
L. Heyne, J. Rhensius, ${ }^{*}$ D. Ilgaz, A. Bisig, U. Rüdiger, and M. Kläui ${ }^{\dagger}$ \\ Fachbereich Physik, Universität Konstanz, Universitätsstrasse 10, D-78457 Konstanz, Germany \\ L. Joly ${ }^{\ddagger}$ and F. Nolting \\ Swiss Light Source, Paul Scherrer Institut, 5232 Villigen PSI, Switzerland \\ L. J. Heyderman \\ Laboratory for Micro- and Nanotechnology, Paul Scherrer Institut, 5232 Villigen PSI, Switzerland
}

J. U. Thiele

Recording Media Operations, Seagate, Fremont, 94538 California, USA

F. Kronast

Helmholtz-Zentrum Berlin für Materialien und Energie GmbH, 12489 Berlin, Germany

(Received 17 June 2010; published 25 October 2010)

\begin{abstract}
We use a pump-probe photoemission electron microscopy technique to image the displacement of vortex cores in Permalloy discs due to the spin-torque effect during current pulse injection. Exploiting the distinctly different symmetries of the spin torques and the Oersted-field torque with respect to the vortex spin structure we determine the torques unambiguously, and we quantify the amplitude of the strongly debated nonadiabatic spin torque. The nonadiabaticity parameter is found to be $\beta=0.15 \pm 0.07$, which is more than an order of magnitude larger than the damping constant $\alpha$, pointing to strong nonadiabatic transport across the high magnetization gradient vortex spin structures.
\end{abstract}

PACS numbers: 75.76.+j, 72.25.Ba, 75.70.Kw, 75.78.Fg

For the development of novel applications [1], the injection of spin-polarized electrons is believed to be a viable alternative approach to manipulate magnetization. Spin currents interact with the magnetization via the adiabatic and nonadiabatic spin torque, that result for instance in domain wall and vortex core (VC) motion [2,3]. To utilize this effect, an in-depth understanding of the torque terms needs to be developed, which has previously been hampered by the difficulty of separating the spin-torque terms and the Oersted-field effect.

The torque terms leading to spin dynamics are described theoretically by the extended Landau-Lifshitz and Gilbert equation including adiabatic and nonadiabatic spin-torque terms $[4,5]$ to account for the interaction of the magnetization with spin-polarized currents

$$
\begin{aligned}
\dot{\vec{m}}= & \gamma_{0} \vec{H} \times \vec{m}+\alpha \vec{m} \times \dot{\vec{m}} \\
& -[\vec{u} \cdot \vec{\nabla}] \vec{m}+\beta \vec{m} \times([\vec{u} \cdot \vec{\nabla}] \vec{m}) .
\end{aligned}
$$

The first two terms describe the damped precession of the magnetization $\vec{m}$ around the effective field $\vec{H}$ with $\alpha$ denoting the damping constant. The third term describes the adiabatic angular momentum transfer from the spinpolarized conduction electrons to the local magnetization, with $\vec{u}$ being proportional to the current density $\vec{j}$. The socalled nonadiabatic fourth term arises from spin relaxation processes $[5,6]$ and from nonadiabatic transport across spin structures with large magnetization gradients $[4,5,7]$.
While the adiabatic spin-torque term is theoretically well understood, the contribution and the size of the nonadiabatic term and, in particular, the value of the nonadiabaticity parameter $\beta$, that governs for instance the domain wall velocity and thus device performance, are highly controversial. Some theoretical work relates $\beta$ directly to the damping constant $\alpha$ [8], whereas others predict it to depend on the local magnetization gradient [7] or to have additional contributions from spin flip and spin orbit effects [6].

Since the Oersted field generated by the current acts on the magnetization as well, this needs to be distinguished from the pure spin-torque contribution in order to unambiguously deduce the spin-torque terms, which has previously not been possible.

Experimentally it was found that injected spin-polarized electrons can displace a magnetic domain wall in the direction of the electron flow [2,3,9]. By studying vortex wall displacement, resonant depinning, domain wall oscillations, and the onset of domain wall motion in Permalloy (Py) wires, values for $\beta$ were estimated indirectly, which range from $\beta \approx \alpha \approx 0.01$ to $\beta=8 \alpha$ and even $\beta \approx 4$ [10-13]. The large variation and the strong assumptions and simplifications used in the models to extract a value for $\beta$ show that a reliable determination of an absolute value for $\beta$ is not straight forward in the wire geometry.

An alternative geometry are discs in the vortex state, where the current pulse induced VC displacement is not 
influenced by edge roughness and potentially the displacement can be directly correlated to the spin-torque. The magnetization of a vortex curls around the $\mathrm{VC}$ either clockwise or counterclockwise which is referred to as the vortex chirality $c= \pm 1$. Figure 1(a) shows a simulation of a counterclockwise vortex $(c=-1)$. In the center, the magnetization tilts out-of-plane to reduce the exchange energy. The direction of the moments in the VC is described by its polarity $p=+1$ or -1 (up or down). In the past only ac-current injection was studied [14], which did not allow for a determination of $\beta$.

In this Letter we use long quasi-dc-current pulses to displace vortex cores in a disc structure quasistatically. We exploit the distinctly different symmetries of the spin torques and the Oersted-field torque to obtain a robust measure of the torques. By imaging the current-induced VC displacement using photoemission electron microscopy, the absolute position of the VC could be determined allowing for a quantification of the amplitude of the strongly debated nonadiabatic spin torque in the high magnetization gradient vortex structures.

To deduce the nonadiabaticity from the core displacement, we first theoretically analyze the influence of the different torque terms on the vortex core. We follow Thiele's approach of a rigid vortex structure and a constant current density, which is an adequate description for the small excitations used here [15]. The VC is treated as a quasiparticle with the corresponding potential depending on the confining geometry. This allows for a significant simplification of the Landau-Lifshitz-Gilbert equation. When current is injected, the VC will be displaced [16] and the final shifted state is shown in the simulation in Fig. 1(b). The final displacement of the VC in the $x$ direction $\left(x_{\mathrm{VC}}\right)$ and the $y$ direction $\left(y_{\mathrm{VC}}\right)$ is a direct measure for the two spin torques. In particular, the angle of the displacement direction $\theta$ [Fig. 1(b)] is directly proportional to the nonadiabaticity parameter $\beta$ :

$$
\tan (\theta)=x_{\mathrm{VC}} / y_{\mathrm{VC}}=p 0.5 \ln (2.0 r / \delta) \beta=p f \beta,
$$

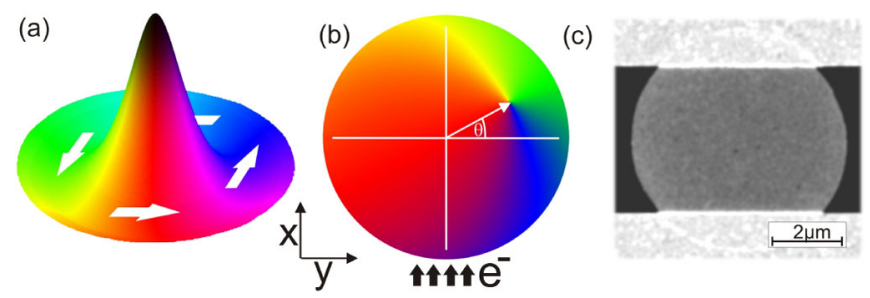

FIG. 1 (color online). Simulation and images of disks in a vortex state. (a) Simulation of a vortex structure. (b) Simulated current-induced VC displacement. The arrow points to the displaced $\mathrm{VC}$ and the angle of the VC displacement direction $\theta$ (angle subtended by the path of the vortex core displacement to the $y$ axis) is shown. (c) SEM image of the $6 \mu \mathrm{m}$ wide and $30 \mathrm{~nm}$ thick Py disk with gold contacts (top and bottom bright contrast). with the disk radius $r$, $\mathrm{VC}$ radius $\delta$, VC polarity $p$ and $f=0.5 \ln (2.0 r / \delta)$ [17]. This simple relation is the key advantage of our approach, since the angle does not depend on any other parameters that are difficult to ascertain and would add to the uncertainty, such as the current density or the damping constant. Furthermore, it is worth noting that nonharmonic contributions relevant for larger displacements [18] do not have an influence on the angle of displacement as long as the confining potential is radially symmetric, so making the relation very robust. Beyond this analytical expression, one can also use micromagnetic simulations to reliably determine the factor $f$ [17]. These simulations have also corroborated the robustness of the method based on Eq. (2) and its independence on the current density.

To determine the value of the nonadiabaticity constant $\beta$, we carry out direct imaging of the current-induced VC displacement in Py disks. The electron beam lithography defined structure is presented in Fig. 1(c), where a scanning electron microscopy (SEM) image of a $30 \mathrm{~nm}$ thick Py disk with a diameter of $6 \mu \mathrm{m}$ on an oxidized $\mathrm{Si}$ substrate is shown. The disk is contacted by $\mathrm{Au}$ pads for current injection at the top and bottom, which are wire bonded on a specially designed sample holder that allows for current injection inside the microscope. We use high resolution x-ray magnetic circular dichroism (XMCD) photoemission electron microscopy (PEEM) [19] to image the initial vortex configuration with a centered $\mathrm{VC}$ as shown in Fig. 2(a). The vortex chirality can be easily determined from the XMCD contrast. The VC polarity is set to the desired direction prior to the imaging by a strong out-ofplane field.

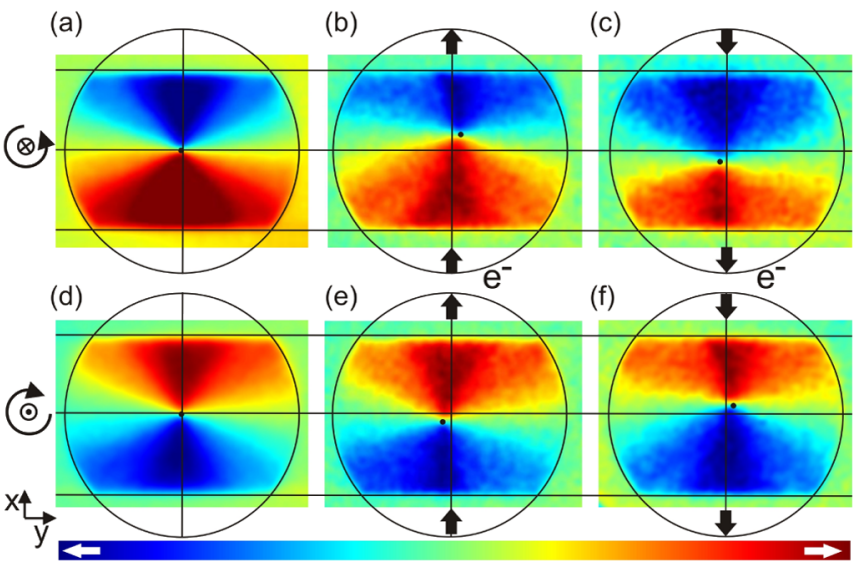

FIG. 2 (color online). Pseudocolor images of current-induced VC displacements. (a) XMCD image of a centered clockwise vortex at zero current. (b) VC displacement for a current density of $j=0.8 \times 10^{12} \mathrm{~A} / \mathrm{m}^{2}$ with the electrons flowing upwards as indicated. (c) VC displacement for the opposite current direction. (d)-(f) show corresponding images for a VC with opposite chirality and polarity. The color bar indicates the direction of the magnetic contrast. 
Joule heating does not allow for continuous current injection, limiting dc-current densities to less than $j=1 \times$ $10^{12} \mathrm{~A} / \mathrm{m}^{2}$ before structural changes set in. To achieve higher current densities, we use $25 \mu$ s long current pulses with a $5 \mu$ s rise and fall time and with a duty cycle of about 1:40 to allow for sufficient cooling time and the relaxation of the vortex core back to its equilibrium position between pulses. The imaging unit of the microscope is gated synchronously to the current pulses to image the structure only while the current is injected. We start the imaging a few microseconds after the pulse plateau when the maximum current density has been reached, so that a stable vortex position is obtained and no more gyrotropic motion occurs. A novel pulsing unit was developed to image the magnetization directly during current injection [20]).

At an injected current density of about $j=0.8 \times$ $10^{12} \mathrm{~A} / \mathrm{m}^{2}$ and with the electrons flowing from bottom to top, the original VC from Fig. 2(a) is displaced resulting in the image shown in (b). Reversing the current direction reverses the $\mathrm{VC}$ displacement as shown in (c). The center of the disks where the VC is located at zero current is marked by the black lines. The images in Fig. 2(d)-2(f) correspond to a vortex with reversed chirality and polarity as indicated.

The VC displacement in the $y$ direction perpendicular to the current is in opposite directions for the images (b) and (e) as well as for (c) and (f). Since the current direction is identical for (b) and (e) as well as for (c) and (f) and the VC polarity is reversed, this is in good agreement with the predictions of the adiabatic spin-transfer torque. However, while the current direction is the same for the images (b) and (e) the VC displacement along the current direction ( $x$ direction) has also changed. This is clearly not a spintorque effect but results from the Oersted field which displaces the VC in opposite directions for opposite VC chiralities.

The Oersted field is generated by possible inhomogeneous current flow in the Py disc [17] and current flowing in the gold contacts and across the gold-permalloy interface where the current direction has a nonvanishing out-ofplane component. Both create an additional field in the $y$ direction. This Oersted field will act as an extra force on the $\mathrm{VC}$ in the $x$ direction, since the domain with the magnetization parallel to the Oersted field ( $y$ direction) will increase in size. Thus an extra term is added to Eq. (2) to account for the displacement of the VC in the $x$ direction due to the Oersted field and this term is proportional to the chirality and the magnetic field strength. From the image sequence it is obvious that the Oersted field is not negligible and needs to be taken into account for the $\mathrm{VC}$ displacement in the current direction.

Since the Oersted-field-induced displacement only depends on the chirality, this opens up the unique possibility to eliminate the Oersted-field term by simply averaging the displacement angles $\theta_{ \pm}$for both chiralities. Additionally for opposite polarities, the difference in the displacement directions effectively yields the pure nonadiabatic spin torque term:

$$
\left(\tan \left(\theta_{+}\right)-\tan \left(\theta_{-}\right)\right) / 2=f \beta .
$$

In Fig. 3, the displaced vortex position is shown for different combinations of polarity, chirality and current direction $\left(j=0.5-0.8 \times 10^{12} \mathrm{~A} / \mathrm{m}^{2}\right)$. To allow for an easier comparison between the different displacements, we make use of the symmetry and mirror all $\mathrm{VC}$ displacements into one disk quadrant.

In the inset, the original data are shown and blue and red data points indicate combinations where the difference in displacement is due to the nonadiabatic spin torque. For the bottom left quadrant this translates into $c=+1$ and $p=+1$ and positive current (red squares) and $c=-1$, $p=-1$ and negative current (blue squares). Because of the opposite chirality and opposite current direction, the Oersted-field effect is the same (moving the $\mathrm{VC}$ in the $-x$ direction), and due to the opposite polarity and opposite current direction, the adiabatic spin torque is the same (moving the $\mathrm{VC}$ in the $-y$ direction) and only the nonadiabatic spin torque leads to the difference in displacement direction $(+x$ for positive current and $-x$ for negative current). Note that the Oersted-field effect is larger than the nonadiabatic spin-torque effect and that the Oersted field is found to purely point in the $y$ direction.

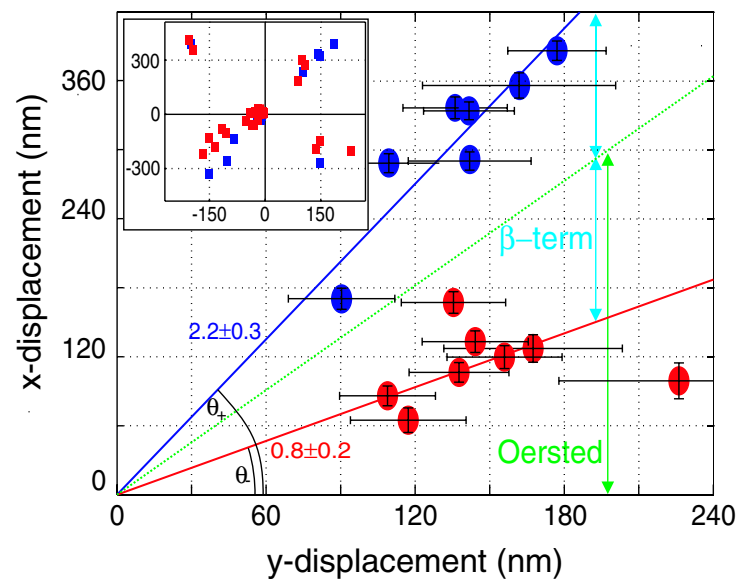

FIG. 3 (color online). Systematic plot of VC displacements. The current-induced VC displacements are plotted for various combinations of polarity, chirality and current direction. The blue and red data points refer to combinations, where the difference in displacement direction is only due to the nonadiabatic spin-torque. Displacement to the lower and left parts of the disk are mirrored into the top right quarter appropriately to allow for an easy comparison between the VC displacements (the inset shows the original data). The value of the fitted slopes for the two chiralities is indicated. The error bars correspond to the accuracy in measuring the VC position. For the VC position in the $x$ direction the error bars are smaller than for the $y$ direction due to the better magnetic contrast in the $x$ direction. 
While the data points show consistent behavior, sometimes strong pinning sites pin the $\mathrm{VC}$, such as seen in the top left quadrant of the inset in Fig. 3. We have carefully checked if a displaced vortex core position is at a pinning site by varying the current density, which should change the position. If, as in the case of the top left quadrant, the $\mathrm{VC}$ is always at the same position independent of the current density, we have excluded these points from the analysis, which results in a lower number of points in Fig. 3 compared to the inset of Fig. 3 .

Averaging these two displacement direction angles $\theta_{ \pm}$ yields the Oersted induced displacement angle $\theta_{\text {Oe }}$, indicated by the green dotted line in Fig. 3. The Oersted-fieldinduced VC displacement corresponds well to a theoretical $\mathrm{VC}$ displacement [21] by a magnetic field of approximately $5 \mathrm{G}$ for $j=0.8 \times 10^{12} \mathrm{~A} / \mathrm{m}^{2}$, which is in line with the field obtained from a numerical evaluation of the Oersted field generated by the current flowing in the Au contacts.

The difference of the slopes for the two VC chiralities now directly yields the nonadiabaticity parameter $\beta$ by evaluating Eqs. (2) and (3). For the disk used in the experiment, we obtain from corresponding micromagnetic simulations $f=4.1 \pm 0.1$, and this results in a value for the nonadiabaticity of $\beta=0.15 \pm 0.07$, that is more than 10 times larger than $\alpha$, which has been determined for our material to be 0.008 [22]. The micromagnetic simulations were performed on disks with a thickness of $30 \mathrm{~nm}$ and varying diameter using the LLG Micromagnetics Simulator [23] with the typical Py parameters: $M_{s}=$ $800 \times 10^{3} \mathrm{~A} / \mathrm{m}, A=1 \times 10^{-12} \mathrm{~J} / \mathrm{m}, P=0.4$. The cell size used was $5 \times 5 \times 30 \mathrm{~nm}^{3}$.

Our results could account for previous observations on current-induced domain wall motion, where higher critical current densities were found for transverse walls than for vortex walls $[3,9]$ while other origins, such as a difference in the pinning strength of the two wall types could of course also play a role. In addition, since the domain wall velocity $\vec{v}$ scales below the Walker breakdown with $\vec{v}=\frac{\beta}{\alpha} \vec{u}$ [4], our finding of a large value of $\beta$ for a vortex core could explain vortex domain wall velocities above $\vec{u}$ that have been observed previously [24]. Given our measured value for $\beta$ and the magnetic parameters for Py [10], this translates below the Walker threshold into a velocity per current ratio of around $200 \mathrm{~m} / \mathrm{s}$ per $10^{11} \mathrm{~A} / \mathrm{m}^{2}$, which means that high wall velocities can be obtained when the Walker breakdown is shifted to higher current densities [25]. Note that recently for vortex walls the large nonadiabaticity was confirmed in an independent measurement [26].

In conclusion, exploiting the symmetry of the system we separate the Oersted-field from the spin torque contribution to the VC displacement and we quantify the values for the spin-torque terms. We measure the nonadiabaticity parameter $\beta$ to be more than an order of magnitude larger than the damping parameter $\alpha$, which bodes well for devices based on domain walls where the performance is governed by the size of the non-adiabatic torque.

The authors acknowledge support by the German Science Foundation (DFG SFB 767, KL1811), the ERC (2007-Stg 208162), the EU (RTN Spinswitch, MRTN CT2006-035327), the Swiss National Science Foundation and the Samsung Advanced Institute of Technology. Part of this work was carried out at the Swiss Light Source and at BESSY.

*Also at LMN, Paul Scherrer Institut, 5232 Villigen, PSI. ${ }^{\dagger}$ Author to whom correspondence should be addressed. Mathias.Klaeui@magnetism.ch

Also at Laboratory for Nanomagnetism and Spin Dynamics, Ecole Polytechnique Fédérale de Lausanne (EPFL), 1015 Lausanne, Switzerland; SwissFEL, Paul Scherrer Institut, 5232 Villigen PSI, Switzerland.

${ }^{\ddagger}$ Present address: IPCMS, UMR7504, 23 rue du Loess BP43, F-67034 Strasbourg cedex 2, France.

[1] S. S. P. Parkin, M. Hayashi, and L. Thomas, Science 320, 190 (2008).

[2] A. Yamaguchi et al., Phys. Rev. Lett. 92, 077205 (2004).

[3] M. Kläui et al., Phys. Rev. Lett. 95, 026601 (2005).

[4] A. Thiaville, Y. Nakatani, J. Miltat, and Y. Suzuki, Europhys. Lett. 69, 990 (2005).

[5] S. Zhang and Z. Li, Phys. Rev. Lett. 93, 127204 (2004).

[6] G. Tatara and P. Entel, Phys. Rev. B 78, 064429 (2008).

[7] J. Xiao, A. Zangwill, and M. D. Stiles, Phys. Rev. B 73, 054428 (2006).

[8] S. E. Barnes and S. Maekawa, Phys. Rev. Lett. 95, 107204 (2005).

[9] L. Heyne et al., Phys. Rev. Lett. 100, 066603 (2008).

[10] G. Meier et al., Phys. Rev. Lett. 98, 187202 (2007).

[11] R. Moriya et al., Nature Phys. 4, 368 (2008).

[12] L. Thomas et al., Nature (London) 443, 197 (2006).

[13] E. Martinez, L. Lopez-Diaz, O. Alejos, and L. Torres, Phys. Rev. B 77, 144417 (2008).

[14] M. Bolte et al., Phys. Rev. Lett. 100, 176601 (2008).

[15] A. A. Thiele, Phys. Rev. Lett. 30, 230 (1973).

[16] J. Shibata, Y. Nakatani, G. Tatara, H. Kohno, and Y. Otani, Phys. Rev. B 73, 020403 (2006).

[17] B. Krüger et al., Phys. Rev. Lett. 104, 077201 (2010).

[18] D. Bedau et al., Phys. Rev. Lett. 101, 256602 (2008).

[19] J. Stöhr et al., Science 259, 658 (1993).

[20] L. Heyne et al., arXiv:1007.5005[Rev. Sci. Instrum. (to be published)].

[21] K. Y. Guslienko et al., Appl. Phys. Lett. 78, 3848 (2001).

[22] J. Walowski et al., Phys. Rev. Lett. 101, 237401 (2008).

[23] LLG Micromagnetics Simulator, http://llgmicro.home .mindspring.com.

[24] M. Hayashi et al., Phys. Rev. Lett. 98, 037204 (2007).

[25] J.-Y. Lee et al., Appl. Phys. Lett. 91, 122513 (2007).

[26] M. Eltschka et al., Phys. Rev. Lett. 105, 056601 (2010). 